\title{
Caracterização da tuberculose em portadores de HIV/AIDS em um serviço de referência de Mato Grosso do Sul
}

\author{
Characterization of tuberculosis among HIV/AIDS patients at a \\ referral center in Mato Grosso do Sul
}

\author{
Maria de Fátima Meinberg Cheade ${ }^{1}$, Maria Lúcia Ivo', \\ Pedro Henrique Guimarães da Silva Siqueira ${ }^{1}$, \\ Robson Gomes de Sá ${ }^{1}$ e Michael Robin Honer ${ }^{2}$
}

\begin{abstract}
RESUMO
Investigou-se a tuberculose quanto à apresentação clínica, desfecho de tratamento e perfil sociodemográfico dos infectados pelo vírus da imunodeficiência humana atendidos em 2003-2005 em um serviço de referência sulmatogrossense. Analisaram-se 66 prontuários de pacientes maiores de 14 anos e informações do Sistema de Informação Nacional de Agravos de Notificação para Tuberculose e do Sistema de Informações de Mortalidade. Predominaram indivíduos do sexo masculino, cor branca, pouca escolaridade e procedência do meio urbano. Identificou-se incremento da apresentação clínica extrapulmonar e sua relação com o comprometimento imunológico. Sobressaíram-se como formas de encerramento da tuberculose a cura (alcançada com acompanhamento mais longo que o previsto) e o óbito (de seis pacientes no início do tratamento da tuberculose). Observaram-se lacunas de preenchimento nas notificações de tuberculose e nos prontuários. Detectou-se a necessidade de diagnosticar precocemente a tuberculose em soropositivos para HIV, de aperfeiçoar os registros nos prontuários e de acompanhar os casos além do período recomendado, por alteração da evolução clínica da tuberculose em co-morbidade com a infecção pelo vírus da imunodeficiência adquirida.
\end{abstract}

Palavras-chaves: Tuberculose. Vírus da imunodeficiência humana. Síndrome da imunodeficiência adquirida. Co-infecção tuberculose-HIV.

\begin{abstract}
Tuberculosis was investigated regarding its clinical presentation, treatment outcome and sociodemographic profile among HIV patients attended at a referral center in Mato Grosso do Sul, in 2003-2005. Sixty-six medical files on patients over 14 years of age and data from the Brazilian National Information System for Notifiable Diseases relating to tuberculosis and from the Mortality Information System were analyzed. Most of the patients were male, white, of low schooling level and from urban areas. Increased extrapulmonary clinical presentation was found and it correlated with the degree of immunological competence. The main reasons for ceasing treatment were cure (reached after longer-than-expected follow-up) and death (of six patients at the beginning of the tuberculosis treatment). Information gaps were found in the tuberculosis notification records and medical files. The study revealed the need for early diagnosis of tuberculosis among HIV-positive patients, improvements in medical records and follow-up beyond the recommended duration, because of changes to the clinical evolution of tuberculosis in cases of comorbidity with HIV.
\end{abstract}

Key-words: Tuberculosis. Human immunodeficiency virus. Acquired immunodeficiency syndrome. Tuberculosis-HIV coinfection.

A epidemia de infecção pelo vírus da imunodeficiência humana (HIV) constitui fator em potencial para a transformação da tuberculose de doença endêmica em epidêmica, em todo o mundo. A infecção por HIV pode ser considerada um dos principais fatores de risco para que indivíduos infectados por Mycobacterium tuberculosis desenvolvam tuberculose doença ${ }^{8}$.

1. Departamento de Enfermagem Universidade Federal de Mato Grosso do Sul, Campo Grande, MS. 2. Universidade Católica Dom Bosco, Campo Grande, MS. Endereço para correspondência: Dra. Maria de Fátima Meinberg Cheade. Departamento de Enfermagem/UFMS. Rua José Fragelli 98, 79009-410 Campo Grande, MS.

Tel: 5567 3382-8487.

e-mail: mdfcheade@uol.com.br

Recebido para publicação em 20/05/2008

Aceito em 05/03/2009
Dos indivíduos infectados por Mycobacterium tuberculosis, mas não por HIV, 90\% não chegarão a apresentar tuberculose ao longo da vida (indivíduos assintomáticos). A possibilidade de que estes indivíduos assintomáticos evoluam para doentes diminui progressivamente com o tempo após o contato inicial com o bacilo ${ }^{8}$.

A soropositividade para HIV incrementa a suscetibilidade a infecção por Mycobacterium tuberculosis e o risco de progressão para doença tuberculose. Tanto na infecção recente quanto na latente, o risco aumenta à medida que a imunossupressão se estabelece $^{719}$. Dessa forma, a tuberculose pode ocorrer em qualquer fase da infecção por HIV ${ }^{8}$ na fase assintomática ou quando já estabelecida a síndrome da imunodeficiência adquirida (AIDS).

0 risco de que um indivíduo não-infectado por HIV desenvolva tuberculose ao longo da vida é de 5\% a 10\%, alcançando 50\% entre os infectados por HIV ${ }^{110}$. Dentre os soropositivos para HIV e 
portadores de infecção latente por Mycobacterium tuberculosis, o risco aumenta 20 vezes em relação aos soronegativos para HIV $^{19}$.

0 aumento da doença tuberculose em portadores de HIV/AIDS impõe sobrecarga aos serviços de saúde, expondo as deficiências que existem nos programas de controle da tuberculose, e apresenta desafios aos profissionais de saúde na definição do diagnóstico, na avaliação e no tratamento, devido às modificações da tuberculose nesses pacientes, desde sua sintomatologia e evolução clínica até a resposta ao tratamento preconizado ${ }^{19}$.

0 propósito deste estudo foi caracterizar, quanto à apresentação clínica, as formas de encerramento e o perfil sociodemográfico nos casos de tuberculose em pacientes portadores de HIV/AIDS atendidos em um serviço de referência de doenças infecto-contagiosas em Mato Grosso do Sul.

\section{MATERIAL E MÉTODOS}

Estudou-se uma série de casos, coletando-se dados secundários em prontuários clínicos de pacientes maiores de 14 anos portadores de HIV/AIDS acometidos pela doença tuberculose, em diferentes formas de apresentação: pulmonar, extrapulmonar e pulmonar e extrapulmonar também denominada de mista, atendidos durante 0 período de primeiro de janeiro 2003 a 31 de dezembro de 2005, no Hospital Universitário Maria Aparecida Pedrossian, da Universidade Federal de Mato Grosso do Sul (HU-UFMS).

Foram incluídos os casos de tuberculose em pacientes soropositivos para HIV em que havia registros dos atendimentos aos pacientes desde a confirmação do diagnóstico da tuberculose até o momento do encerramento do tratamento, sendo excluídos os prontuários em que os registros não atendessem integralmente estas especificações.

0 diagnóstico de doença tuberculose e a presença de HIV/AIDS foram identificados por meio da história clínica dos pacientes, em anotações dos profissionais de saúde e dos resultados de exames laboratoriais registrados nos prontuários clínicos, tais como pesquisa de BAAR e cultura de escarro, pesquisa de BAAR e cultura de outros tecidos e materiais e histopatológico de tecido, radiografia de tórax. O diagnóstico de tuberculose seguiu os critérios recomendados pelo Guia de Vigilância Epidemiológica ${ }^{6}$. Os pacientes soropositivos para HIV foram incluídos independentemente do estágio de evolução dessa infecção.

Elaborou-se um formulário para identificação das seguintes variáveis no período da co-infecção por tuberculose e HIV: perfil sociodemográfico, dados clínicos da doença tuberculose, datas e resultados dos exames laboratoriais, condição imunológica inicial dos pacientes com tuberculose e formas de encerramento do tratamento da tuberculose.

A condição imunológica se baseou na contagem de linfócitos $\mathrm{T} \mathrm{CD}^{+}$, para a qual a coleta de sangue foi realizada em até um ano antes do início de tratamento da tuberculose.

Do ponto de vista clínico, as concentrações séricas de linfócitos $\mathrm{T} \mathrm{CD}^{+}{ }^{+}$foram agrupadas de modo a delimitar duas fases de comprometimento imunológico, segundo a classificação utilizada por Kritski e cols ${ }^{10}$ denominadas de:

Fase precoce do comprometimento imunológico: pacientes soropositivos para HIV com concentração sérica de linfócitos T $\mathrm{CD}^{+}$maior ou igual a 200 células $/ \mathrm{mm}^{3}$ ou de linfócitos totais maior ou igual a 1.000 células $/ \mathrm{mm}^{3}$.

Fase avançada de comprometimento imunológico: pacientes soropositivos para HIV com concentração sérica de linfócitos T $\mathrm{CD}^{+}$menor que 200 células $/ \mathrm{mm}^{3}$ ou de linfócitos totais séricos menor que 1.000 células $/ \mathrm{mm}^{3}$.

As formas de encerramento foram determinadas segundo as informações dos prontuários, complementadas e comparadas com informações do Sistema de Informação Nacional de Agravos de Notificação para Tuberculose (SINAN-TB) e do Sistema de Informações de Mortalidade (SIM).

0 projeto de pesquisa foi submetido à avaliação do Comitê de Ética da Universidade Federal de Mato Grosso do Sul, obtendo parecer favorável. Foram atendidos os pressupostos éticos de pesquisa em seres humanos e mantido o sigilo das informações coletadas.

A apresentação descritiva dos dados foi feita mediante a tabulação numérica e por frequiência, utilizando o software Microsoft Excel.

\section{RESULTADOS}

Entre os 66 pacientes cujos prontuários foram analisados, o diagnóstico de tuberculose e a detecção de HIV/AIDS ocorreram em datas próximas, sendo a soropositividade por HIV diagnosticada anteriormente ao diagnóstico da tuberculose, variando em intervalo de até um mês em 22 (33\%) casos e em intervalo de até um ano em 44 (66\%) (Tabela 1).

\section{TABELA 1}

Intervalo entre o diagnóstico de tuberculose e a detecção de HIV/AIDS em pacientes atendidos no Hospital Universitário, Universidade Federal de Mato Grosso do Sul, Campo Grande, MS, 2003-2005.

\begin{tabular}{lrr}
\hline Intervalo entre diagnóstico & & \\
de tuberculose e detecção de HIV/AIDS & Número & Porcentagem \\
\hline $0-1$ mês & 22 & 33,3 \\
1 mês -I 1 ano & 22 & 33,3 \\
1 ano - I 5 anos & 10 & 15,2 \\
5 anos - I 10 anos & 8 & 12,1 \\
$>10$ anos & 4 & 6,1 \\
\hline Total & 66 & $\mathbf{1 0 0 , 0}$ \\
\hline
\end{tabular}

No perfil sociodemográfico dos pacientes, predominaram sexo masculino, cor da pele branca e residência no município de Campo Grande, MS. Dois pacientes viviam no meio rural e 64 no meio urbano, cinco dos quais eram reclusos em instituições (presídio ou casa de abrigo), apresentados na Tabela 2.

Verificou-se que $48(72,7 \%)$ dispunham de até oito anos de estudo (ensino fundamental), sete $(10,6 \%$ ) haviam alcançado o ensino médio e um $(1,5 \%)$ o ensino superior. Quanto à idade, predominou a faixa de 25 a 34 anos, com idade mínima de 21 e máxima de 63 anos. 
TABELA 2

Perfil sociodemográfico de pacientes portadores de HIV/AIDS acometidos de tuberculose atendidos no Hospital Universitário, Universidade Federal de Mato Grosso do Sul, Campo Grande, MS, 2003-2005.

\begin{tabular}{|c|c|c|}
\hline Descrição & Número & Porcentagem \\
\hline \multicolumn{3}{|l|}{ Sexo } \\
\hline masculino & 49 & 74,2 \\
\hline feminino & 17 & 25,8 \\
\hline \multicolumn{3}{|l|}{ Cor da pele } \\
\hline branca & 45 & 68,1 \\
\hline negra & 3 & 4,5 \\
\hline parda & 15 & 22,7 \\
\hline sem informação & 3 & 4,5 \\
\hline \multicolumn{3}{|l|}{ Residência } \\
\hline interior de Mato Grosso do Sul & 11 & 16,6 \\
\hline capital de Mato Grosso do Sul & 53 & 80,3 \\
\hline municípios de Mato Grosso & 2 & 3,0 \\
\hline \multicolumn{3}{|l|}{ Ambiente de moradia } \\
\hline área urbana & 59 & 89,9 \\
\hline área rural & 2 & 3,0 \\
\hline instituições & 5 & 7,6 \\
\hline \multicolumn{3}{|l|}{ Escolaridade } \\
\hline ensino fundamental & 48 & 72,7 \\
\hline ensino médio & 7 & 10,6 \\
\hline ensino superior & 1 & 1,5 \\
\hline não informado & 10 & 15,1 \\
\hline \multicolumn{3}{|l|}{ Idade (anos) } \\
\hline 15 a 24 & 5 & 7,6 \\
\hline 25 a 34 & 29 & 43,9 \\
\hline 35 a 44 & 18 & 27,3 \\
\hline 45 a 54 & 9 & 13,6 \\
\hline$\geq 55$ & 5 & 7,6 \\
\hline
\end{tabular}

Quanto à apresentação clínica da tuberculose, houve localização pulmonar em 33 (50\%) dos casos, extrapulmonar em 21 (31,8\%) e mista (pulmonar e extrapulmonar simultâneas) em 12 (18,2\%). Nas apresentações clínicas extrapulmonares, predominou a ganglionar periférica com 18 (54,5\%) casos (Tabela 3).

\section{TABELA 3}

Apresentação clínica da tuberculose e distribuição da tuberculose extrapulmonar em pacientes portadores de HIV/AIDS atendidos no Hospital Universitário, Universidade Federal de Mato Grosso do Sul, Campo Grande, MS, 2003-2005.

\begin{tabular}{lrr}
\hline Apresentação clínica da tuberculose & Número & Porcentagem \\
\hline Pulmonar & 33 & 50,0 \\
Extrapulmonar & 21 & 31,8 \\
Mista (pulmonar e extrapulmonar) & 12 & 18,2 \\
\hline Total & $\mathbf{6 6}$ & $\mathbf{1 0 0 , 0}$ \\
\hline
\end{tabular}

Tuberculose extrapulmonar*

\begin{tabular}{lrr} 
Pleural & 3 & 9,1 \\
Ganglionar periférica & 18 & 54,5 \\
Miliar & 4 & 12,1 \\
Meningoencefálica & 6 & 18,2 \\
Outras & 2 & 6,1 \\
\hline Total & $\mathbf{3 3}$ & $\mathbf{1 0 0 , 0}$
\end{tabular}

*Compreende os casos extrapulmonares e as formas extrapulmonares da apresentação mista.
Para avaliação da condição imunológica dos pacientes, dispôsse de apenas 30 exames nos prontuários, que revelaram que 15 pacientes se encontravam em comprometimento imunológico avançado (Tabela 4).

Quanto à distribuição da apresentação clínica da tuberculose e sua relação com a condição imunológica inicial, verificou-se que nos pacientes com contagem de linfócitos T $\mathrm{CD}^{+}$inferior a 200 células/ $/ \mathrm{mm}^{3}$ predominou a apresentação pulmonar, embora a extrapulmonar e a mista agrupadas perfizessem o mesmo número de casos que a pulmonar. A apresentação extrapulmonar predominou em pacientes com comprometimento imunológico.

Nos encerramentos de tratamento da tuberculose, houve maior número de curas com adoção dos esquemas terapêuticos disponíveis, com predomínio do Esquema 1 (Tabela 5).

Dentre os casos de encerramento por abandono, um paciente era procedente de abrigo e o outro não concluiu o tratamento, apesar dos registros de reinícios em um período de três a quatro anos. Esse paciente, entretanto, continuou a receber tratamento para tuberculose e HIV no mesmo serviço.

Em 53\% dos casos de tuberculose estudados, houve registros de internação no HU-UFMS em diferentes momentos: no atendimento ao paciente, na investigação do diagnóstico e também durante e no final do tratamento da tuberculose.

Dos 25 pacientes em que a contagem de linfócitos $\mathrm{T} \mathrm{CD} 4^{+}$foi inferior ou igual a 350 células $/ \mathrm{mm}^{3}$, 19 foram internados.

Dos casos analisados, 17 (25,7\%) pacientes faleceram após o início do tratamento da tuberculose, sendo esta registrada como causa secundária do óbito e, ainda, os encerramentos por óbito ocorreram nos seis primeiros meses de tratamento da tuberculose (Tabela 5).

\section{TABELA 4}

Apresentação clínica da tuberculose segundo o comprometimento imunológico de pacientes portadores de HIV/AIDS atendidos no Hospital Universitário, Universidade Federal de Mato Grosso do Sul, Campo Grande, MS, 2003-2005.

\begin{tabular}{|c|c|c|c|c|c|}
\hline \multirow[b]{3}{*}{ Apresentação clínica } & \multicolumn{4}{|c|}{ Comprometimento imunológico* } & \multirow[b]{3}{*}{ Total } \\
\hline & \multicolumn{2}{|c|}{ fase avançada } & \multicolumn{2}{|c|}{ fase precoce } & \\
\hline & $\leq 100$ & $>100 \mathrm{e} \leq 200$ & $>200 \mathrm{e} \leq 350$ & $>350$ & \\
\hline Pulmonar & 4 & 3 & 6 & 3 & 16 \\
\hline Extrapulmonar & 5 & 1 & 4 & 1 & 11 \\
\hline Mista $^{* * *}$ & 2 & 0 & 0 & 1 & 3 \\
\hline Total & 11 & 4 & 10 & 5 & 30 \\
\hline
\end{tabular}

*Contagem de linfócitos $\mathrm{T} \mathrm{CD}^{+}{ }^{+}\left(\right.$células $\left./ \mathrm{mm}^{3}\right)$ realizada no máximo um ano antes do início do tratamento de tuberculose, ${ }^{* *}$ Pulmonar e extrapulmonar.

\section{TABELA 5}

Formas de encerramento de tratamento antituberculose, segundo tipo de terapia, em pacientes portadores de HIV/AIDS atendidos no Hospital Universitário, Universidade Federal de Mato Grosso do Sul, Campo Grande, MS, 2003-2005.

\begin{tabular}{lcccccc}
\hline \multirow{2}{*}{$\begin{array}{l}\text { antituberculose } \\
\text { nyyyynn}\end{array}$} & cura & abandono & óbito & transferência & SI & Total \\
\hline Esquema 1 & 37 & 2 & 3 & 5 & 2 & 49 \\
Esquema 1R & 4 & 0 & 2 & 0 & 1 & 7 \\
Esquema 1+1R & 2 & 0 & 0 & 2 & 0 & 4 \\
Esquema 2 & 3 & 0 & 1 & 0 & 2 & 6 \\
\hline Total & $\mathbf{4 6}$ & $\mathbf{2}$ & $\mathbf{6}$ & 7 & $\mathbf{5}$ & $\mathbf{6 6}$ \\
\hline
\end{tabular}

SI: sem informação, no prontuário, da data final da terapia medicamentosa. Esquema 1+1R: casos em que o Esquema 1, inicialmente adotado, foi substituído pelo Esquema $1 \mathrm{R}$ no reinício do tratamento. 


\section{DISCUSSÃo}

A tuberculose é a única das infecções oportunistas características da AIDS que é transmissível para outros indivíduos, e seu diagnóstico e tratamento precoce podem bloquear sua transmissão à população em geral'.

Neste estudo, constatou-se a ocorrência da doença tuberculose em 13,5\% dos pacientes portadores de HIV/AIDS acompanhados pelo serviço de doenças infecto-parasitárias do HU-UFMS no período de 2003-2005.

No Brasil, a expectativa para a taxa de co-infecção por Mycobacterium tuberculosis em infectados por HIV é de 3\% a 4\%, mesmo com dados subestimados. Na Região Sudeste, onde a prevalência de infecção por HIV é a maior do país, identificam-se em média, para os atendimentos em ambulatório a pacientes de tuberculose, taxas de $10 \%$ a $15 \%$ de co-infecção por Mycobacterium tuberculosis em portadores de HIV, que sobem para $25 \%$ a $30 \%$ nos casos atendidos em unidades hospitalares ${ }^{10}$.

Em Mato Grosso do Sul, em 2005, dos 1172 casos de tuberculose notificados ao SINAN-TB, 695 (59,3\%) foram testados para HIV, verificando-se prevalência mínima de $7,7 \%$ de coinfecção (prevalência de positividade de HIV entre os casos de tuberculose) e máxima de 12,9\% (prevalência de positividade de HIV entre os pacientes testados) ${ }^{22}$.

Na capital do Estado, Campo Grande, na série histórica de 2000 a 2005, a prevalência mínima de tuberculose em pacientes infectados por HIV variou de 8,5\% (18/212) em 2000 a 16,6\% (48/289) em 2005. A prevalência máxima, por sua vez, variou de 18,2\% (35/192) em 2004, a 37,9\% (36/95), em 2002 ${ }^{14}$.

0 fato dos diagnósticos de tuberculose e de presença de HIV/ AIDS ocorrerem em período próximo demonstra a busca ativa do diagnóstico da tuberculose em pacientes soropositivos para HIV pelo serviço, e ao mesmo tempo, reforça a necessidade de aconselhamento para a realização de teste anti-HIV em caráter voluntário em pacientes com tuberculose, qualquer que seja a forma clínica dessa doença, na busca do diagnóstico precoce dessa infecção, como já recomendam o Ministério da Saúde e as diretrizes do tratamento de tuberculose preconizadas no II Consenso de Pneumologiá.

A sorologia anti-HIV em pacientes com tuberculose é importante, dada a alta prevalência da co-infecção por Mycobacterium tuberculosis e HIV e por não ser conclusiva a identificação dos co-infectados baseada apenas na história clínica. Recomenda-se também o diagnóstico precoce dessa co-infecção, devido à mútua interação entre HIV e Mycobacterium tuberculosis e às implicações terapêuticas do tratamento de tuberculose e de HIV/AIDS, predispondo ao agravamento clínico ${ }^{1}$.

Para maior efetividade do sistema de vigilância epidemiológica da tuberculose, recomenda-se promover o diagnóstico de coinfecção por Mycobacterium tuberculosis e HIV para que se possa precisar a magnitude daquele agravo com base na proporção dos testes de sorologia para HIV realizados, aumentando-se com isso a exatidão dos indicadores para seu acompanhamento 9 .
No presente estudo, constatou-se predomínio do sexo masculino, na razão aproximada de 2,9 homens para cada mulher. Esse predomínio, já apontado em estudos sobre tuberculose ${ }^{14}$, acompanha a tendência dessa doença em Mato Grosso do Sul, segundo informações do SINAN-TB' ${ }^{22}$.

O predomínio do sexo masculino, tanto na tuberculose como na AIDS, também é descrito em outros estudos em diferentes localidades do Brasil desde o início da década de $1990^{61213171825}$, confirmando a população masculina como mais vulnerável e prevalente para co-infecção por Mycobacterium tuberculosis e HIV.

Ao mesmo tempo, observa-se aumento dos índices de acometimento de tuberculose em mulheres, o que provavelmente é influenciado pela co-infecção por Mycobacterium tuberculosis e HIV, cuja alteração no estado de São Paulo ${ }^{25}$ é atribuída a mudanças epidemiológicas da AIDS.

Quanto à cor da pele, houve predomínio de pacientes brancos, embora na coleta dessa informação tenha se verificado o maior número de divergências nas anotações dos prontuários, possivelmente pela dificuldade em definir essa variável, dadas as características étnicas da população sulmatogrossense, em grande parte de descendência indígena do sul do estado e dos países vizinhos (Paraguai e Bolívia).

A faixa etária com maior ocorrência, de 25 a 34 anos, seguida da faixa de 35 a 44 anos, revela que os pacientes são adultos jovens, em plena fase produtiva da vida profissional, o que traz repercussões sociais para paciente, família e sociedade. Esses dados são coincidentes com os da literatura ${ }^{13} 1516$ 17, os quais confirmam tais faixas etárias como alvos das epidemias de AIDS e de tuberculose.

Outra tendência verificada foi a existência de pacientes na faixa etária de 45 a 54 anos, bem como casos de tuberculose em pacientes com mais de 55 anos, elevando a faixa etária daqueles acometidos por tuberculose e AIDS. Tal achado está em concordância com o expresso em documentos do Programa Nacional de Doenças Sexualmente Transmissíveis e AIDS ${ }^{15}$, que apontam tendência de crescimento nos casos de AIDS em homens de 40 a 59 anos.

o predomínio de residentes em Campo Grande permite a hipótese de que pacientes de outras localidades optam por transferir-se a esse município por disporem nele de serviços de referência que facultam melhor acesso a atendimentos de maior complexidade.

A pouca escolaridade dos pacientes analisados, que dispunham em sua maioria de até oito anos de estudo, pode refletir-se em suas possibilidades profissionais, restringindo-os a condições desfavoráveis de vida e emprego, mantendo seu estado de pobreza. É exatamente nessa população que a incidência da infecção por HIV é elevada, fomentando a manutenção de condições sociais desfavoráveis e um ambiente propício ao incremento da prevalência de tuberculose.

0 fenômeno da pauperização de pacientes acometidos por AIDS no Brasil desde 1991 é frisado em estudos ${ }^{32}$ que constataram que a co-infecção por Mycobacterium tuberculosis e HIV acomete majoritariamente indivíduos que vivem em 
condições socioeconômicas desfavoráveis. Lima ${ }^{13}$ salienta em seus achados que a escolaridade inferior a oito anos e a baixa qualificação profissional são fatores predisponentes a infecções e à não-adesão ao tratamento.

No presente estudo, a apresentação clínica da tuberculose encontrada com maior frequiência foi a pulmonar. As apresentações extrapulmonares foram, porém, maiores que o esperado, corroborando estudos realizados em outras localidades 2316171825 .

Das apresentações clínicas extrapulmonares, a ganglionar periférica foi a de maior frequiência. Semelhante situação foi relatada por Boffo e cols ${ }^{2}$, que constataram $61,3 \%$ de apresentações pulmonares, 32,3\% de extrapulmonares e 6,5\% de mistas.

A tuberculose pode se tornar ativa em qualquer fase da evolução da infecção por HIV, mas em pacientes em estado avançado de comprometimento imunológico tem como forma de apresentação mais comum a extrapulmonar ${ }^{3}$.

Neste trabalho, em 30 prontuários que informavam as contagens linfocitárias anteriores ao início do tratamento de tuberculose, identificaram-se 25 pacientes em estágio de imunossupressão, com níveis de linfócitos T CD $4^{+}$de até 350 células $/ \mathrm{mm}^{3}$, o que caracteriza uma das condições imunológicas definidoras de AIDS $^{21}$, condição que pôde ser confirmada nas notificações de AIDS desses pacientes no SINAN-AIDS. Em 15 desses 30 prontuários constavam níveis de linfócitos T $\mathrm{CD}^{+}$de até 200 células $/ \mathrm{mm}^{3}$, revelando fase avançada de comprometimento imunológico, o que demonstra a gravidade clínica desses casos.

Houve coincidência com os achados de outros estudos ${ }^{31024}$, em que se confirmou que a modificação da apresentação clínica da tuberculose em pacientes infectados por HIV relaciona-se com a imunossupressão causada por esse vírus, já que na ocorrência de tuberculose sem infecção por HIV as expectativas são de $90 \%$ de casos pulmonares e $10 \%$ de extrapulmonares ${ }^{6}$.

A forma de finalização de tratamento de tuberculose que predominou no presente estudo foi a cura, achado esse que difere dos obtidos em investigações realizadas em municípios do interior de São Paulo ${ }^{317}$.

Nos encerramentos por cura, os prontuários mencionavam tratamentos de nove meses ou mais, o que possivelmente se deve ao agravamento das condições de pacientes co-infectados por HIV, devido às inúmeras intercorrências clínicas e às dificuldades advindas do tratamento simultâneo, que incluem interações medicamentosas. Esse achado aponta a necessidade de acompanhar de maneira diferenciada os pacientes co-infectados, providência essa que pode se estender por períodos prolongados, superiores aos recomendados pelo Programa Nacional de Controle da Tuberculose, de seis a nove meses, para tratamento e encerramento dos casos de tuberculose ${ }^{4}$.

Por outro lado, a ocorrência de óbitos no período inicial do tratamento da tuberculose e o número de internações comprovam a gravidade desses pacientes na época do diagnóstico de tuberculose, além de possivelmente revelarem demora no estabelecimento desse diagnóstico.
O diagnóstico precoce da tuberculose, em pacientes infectados por HIV, e o início de seu tratamento interrompe a evolução da doença, o que predispõem a melhora no estado clínico, com repercussões positivas no prognóstico ${ }^{7}$.

Nos primeiros seis meses de tratamento de tuberculose, a taxa de mortalidade dos pacientes analisados diferiu daquela proveniente das declarações de óbito, mesmo que constassem nos prontuários informações sobre o uso de terapia antituberculose, o que revela subnotificação, achado esse que coincide com os de outros estudos ${ }^{2023}$.

As buscas realizadas nos registros do SINAN-TB mostraram que os casos foram concluídos pelo sistema municipal de saúde, com informações fornecidas pelo HU-UFMS sobre o início do tratamento e a forma de encerramento, aos seis meses e de nove meses para os casos meningite tuberculose, como preconizados para o controle da tuberculose. Nos casos de co-infecção por HIV, porém, a tuberculose tem modificações clínicas acentuadas em sua evolução, que não são rotineiramente informadas ao SINAN-TB.

Verificaram-se falhas no preenchimento dos prontuários clínicos e, ao mesmo tempo, subnotificação ao SINAN-TB, além de falhas no preenchimento das declarações de óbito, que fundamentam o SIM. Os prontuários clínicos e as notificações dos agravos por doenças infecciosas de notificação compulsória são preenchidos com as informações pelas quais são responsáveis todos os profissionais que prestam atendimento ao paciente.

os fatos descritos podem indicar deficiências do monitoramento dos pacientes no controle da tuberculose. São registrados como casos de abandono de tratamento aqueles em que o paciente deixa de retornar ao serviço em até um mês durante o tratamento. Nos prontuários pesquisados, não havia registro de realização de buscas para localização desses pacientes. Um período de seis e de nove meses parecem insuficientes para que se considere como encerrados os casos de tuberculose em infectados por HIV, devido às intercorrências ocasionadas por essa co-morbidade e também pelas reações adversas da terapia medicamentosa da tuberculose e dos anti-retrovirais, requerendo-se portanto uma conduta de monitoramento que difira daquela adotada para pacientes não infectados por HIV.

A mudança do local de atendimento, empreendida por iniciativa do paciente, pode deixar de ser identificada pelo serviço de vigilância em saúde, dificultando, pela falta de atualização das informações, a continuidade dos atendimentos para tuberculose e HIV.

Tem sido apontada ${ }^{11}$ a necessidade de rever as estratégias de acompanhamento dos pacientes com tuberculose, frente ao elevado (38\%) índice de abandono do tratamento e da profilaxia da tuberculose, e também devido à associação entre esse abandono e a não-adesão a tratamento de AIDS.

0 fato de os pacientes portadores de HIV/AIDS estarem vinculados a um serviço de referência, com acompanhamento previamente agendado para a continuidade do tratamento, possibilita-lhes acesso aos atendimentos, garantia do fornecimento de medicamentos e estímulo à adesão às terapias medicamentosas de ambas as patologias, além de retorno regular ao tratamento, em casos de interrupção ou irregularidade. 
Para os serviços de saúde, existem recomendações da Organização Mundial de Saúde, do Ministério da Saúde e da Sociedade Brasileira de Pneumologia ${ }^{45}$ para a integração e estabelecimento de ações conjuntas voltadas ao controle da tuberculose e da AIDS. As ações direcionadas ao controle da tuberculose são de fundamental importância para a efetividade das ações programáticas voltadas a HIV/AIDS. Esses dois serviços devem integrar-se, com estabelecimento formal de fluxos de atendimento, acompanhamento e busca dos casos de tuberculose para controle dessa doença na instituição e com integração ao Sistema Municipal de Saúde.

Concluindo, o perfil sociodemográfico dos pacientes analisados foi coincidente com o encontrado por outros estudos, com predomínio de homens jovens em idade produtiva e com poucos anos de estudo.

Os pacientes eram procedentes, em sua maioria, de Campo Grande e de áreas urbanas.

Houve predomínio de cor de pele branca, embora nos registros feitos nos prontuários pelos profissionais que atenderam os pacientes tenham sido observadas divergências nessa informação.

Os diagnósticos da doença tuberculose e de HIV/AIDS ocorreram em datas próximas ou divergiram em cerca de um ano, o que salienta a necessidade de se oferecer o teste anti-HIV para todos os pacientes com diagnóstico de tuberculose.

A tuberculose pulmonar foi predominante, com incremento das formas extrapulmonares e mistas. Identificou-se modificação das apresentações clínicas da doença em pacientes com a imunossupressão causada por HIV.

Houve alta frequiência de internações nesse grupo, sugerindo que a tuberculose em pacientes portadores de HIV/AIDS constituiu fator colaborador para a hospitalização nas diferentes fases do desenvolvimento da doença tuberculose, a qual, na ausência dessa co-infecção, é de hospitalização muito restrita, sendo suficiente, em geral, o tratamento em ambulatório.

Identificaram-se como formas de encerramento a cura, 0 abandono, o óbito e a transferência, com predomínio dos casos de cura. Seis óbitos ocorreram em até seis meses após o início do tratamento.

Ao se compararem os registros da evolução clínica dos pacientes nos prontuários, no SINAN-TB e no SIM, observaram-se discordâncias, com falhas nas anotações durante 0 acompanhamento desses pacientes.

Conclui-se serem oportunos estudos adicionais, com diferentes bases metodológicas, que permitam aperfeiçoar 0 atendimento aos pacientes com tuberculose e co-infectados por HIV, visando evitar a ocorrência de complicações e a necessidade de internações hospitalares, de modo a incrementar-se o controle da tuberculose no HU-UFMS.

\section{REFERÊNCIAS}

1 Atomiya AN, Uip DE, Leite OHM. Evaluation of disease patterns, treatment and prognosis of tuberculosis in AIDS patient. Brazilian Journal of Infectious Disease 6: 29-39, 2002
2 Boffo MMS, Mattos IG, Ribeiro M0, Oliveira Neto IC. Tuberculose associada à AIDS: características demográficas, clínicas e laboratoriais de pacientes atendidos em um serviço de referência do sul do Brasil. Jornal Brasileiro de Pneumologia 30: 140-146, 2004.

3 Carvalho LGM, Buani AZ, Zöllner MSAC, Scherma AP. Co-infecção por Mycobacterium tuberculosis e vírus da imunodeficiência humana: uma análise epidemiológica em Taubaté (SP). Jornal Brasileiro de Pneumologia 32: 424-429, 2006.

4 Castelo Filho A e colaboradores. II Consenso Brasileiro de Tuberculose: Diretrizes Brasileiras para Tuberculose. Jornal Brasileiro de Pneumologia 30 (supl):S1-S55, 2004.

5 Fundação Nacional de Saúde. Controle da Tuberculose: Uma proposta de integração ensino-serviço. $5^{\text {a }}$ edição. Ministério da Saúde, Rio de Janeiro, 2002.

6 Fundação Nacional da Saúde.Guia de Vigilância Epidemiológica. Secretária de Vigilância em Saúde, Ministério da Saúde, Brasília, 2002.

7 Godfrey-Faussett P, Maher D, Mukadi YD, Nunn P, Perriëns J, Raviglione M. How human immunodeficiency virus voluntary testing can contribute to tuberculosis control. Bulletin of the World Health Organization 80: 939-945, 2002.

8 Harries A, Maher D, Graham S. TB/HIV: a clinical manual. 2 edição. World Health Organization library. Geneva, 2004.

9 Jamal LF, Moherdaui F. Tuberculose e infecção pelo HIV no Brasil: magnitude do problema e estratégias para o controle. Revista de Saúde Pública 41: 104-110, 2007.

10 Kritski A, Conde M, Souza G. Tuberculose do ambulatório à enfermaria. $3^{a}$ edição. Atheneu, São Paulo, 2005.

11 Lana F, Rodrigues F, Diniz M. Adesão ao tratamento/profilaxia de tuberculose associada à infecção HIV/AIDS no Centro de Treinamento e Referência de Doenças Infecciosas e Parasitárias Orestes Diniz. Revista Mineira de Enfermagem 7: 9-13, 2003.

12 Liberato IRO, Albuquerque MFPM, Campelo ARL, Melo HRL. Characteristics of pulmonary tuberculosis in HIV seropositive and seronegative patients in a Northeastern region of Brazil. Revista da Sociedade Brasileira de Medicina Tropical 37: 46-50, 2004.

13 Lima HMM. Adesão ao Tratamento do HIV/AIDS por pacientes com AIDS tuberculose e usuários de Drogas em São Paulo.Tese de Doutorado, Universidade de São Paulo, São Paulo, SP, 2006.

14 Marques M, Cheade MFM, Gazola LH. SINAN na detecção de co-infecção TB-HIV Boletim de Pneumologia Sanitária 14: 135-140, 2006.

15 Ministério da Saúde. Programa Nacional de DST e AIDS. Boletim Epidemiológico. AIDS DST. Ano II - n. 1-01-26 de 2005 semanas epidemiológicas janeiro a junho de 2005. Brasília, 2005.

16 Morimoto AA, Bonametti AM, Morimoto HK, Matsuo T. Soroprevalência da infecção pelo vírus da imunodeficiência humana em pacientes com tuberculose, em Londrina, Paraná. Jornal Brasileiro de Pneumologia 31: 325-331, 2005.

17 Muniz JN, Ruffino-Netto A, Villa TCS, Yamamura M, Arcencio R, Cardozo-Gonzales RI. Aspectos epidemiológicos da co-infecção tuberculose e vírus da imunodeficiência humana em Ribeirão Preto (SP), de 1998 a 2003. Jornal Brasileiro de Pneumologia 32: 529-534, 2006.

18 Pasqualotto AC, Rosa DD, Pereira MCF, Targa-Ferreira RL, Santos BR. Retrospective study of 668 cultures for mycobacteria in a reference hospital for AIDS in Southern Brazil. Brazilian Journal of Infectious Disease 7: 126-128, 2003.

19 Reid A, Scano F, Haileyesus G, Williams B, Dye C, Nunn P, De Cock KM, Hankins C, Miller B, Castro KG. Raviglione MC. Towards universal access to HIV prevention, treatment, care, end support: the role of tuberculosis/HIV collaboration. Lancet Infectious 6: 483-495, 2006

20 Santos AH. Causas múltiplas de morte relacionadas à tuberculose no Estado do Rio de Janeiro entre 1999 e 2001. Jornal Brasileiro de Pneumologia 32: 544-552, 2006.

21 Secretaria de Vigilância em Saúde. Programa Nacional de DST e AIDS. Critérios de definição de casos de AIDS em adultos e crianças. Ministério da Saúde, Brasília: (Séries manuais n⿳0 60), 2004.

22 Secretaria do Estado de Saúde. Dados epidemiológicos do Programa estadual de controle da tuberculose (PCT). SES. Campo Grande, 2007. 
23 Selig L, Belo M, Cunha AJLA, Teixeira EG, Brito R, Luna AL, Trajman A. Óbitos atribuídos à tuberculose no Estado do Rio de Janeiro. Jornal Brasileiro de Pneumologia 30: 335-342, 2004.

24 Silveira JM, Sassi RAM, Oliveira Netto IC, Hetzel JL. Prevalência e fatores associados à tuberculose em pacientes soropositivos para o vírus da imunodeficiência humana em centro de referência para tratamento da síndrome da imunodeficiência adquirida na região sul do Rio Grande do Sul. Jornal Brasileiro de Pneumologia 32: 48-55, 2006.

25 Song ATW, Schout D, Novaes HMD, Goldbaum M. Clinical and epidemiological feature of AIDS/tuberculosis comorbidity. Revista Hospital Clinicas 58: 207-214, 2003 